\title{
Critical coupling for chiral symmetry breaking in QCD motivated models
}

\author{
A. C. Aguilar, A. A. Natale, and R. Rosenfeld \\ Instituto de Física Teórica, Universidade Estadual Paulista, Rua Pamplona 145, 01405-900, São Paulo, SP, Brazil
}

(Received 21 January 2000; revised manuscript received 8 June 2000; published 3 October 2000)

\begin{abstract}
We determine the critical coupling constant above which dynamical chiral symmetry breaking occurs in a class of QCD motivated models where the gluon propagator has an enhanced infrared behavior. Using methods of bifurcation theory we find that the critical value of the coupling constant is always smaller than the one obtained for QCD.
\end{abstract}

PACS number(s): 12.38.Lg, 12.38.Aw, 24.85.+p

\section{INTRODUCTION}

Hadronic physics at low energy is expected to be described by the infrared properties of quantum chromodynamics (QCD), whose nonperturbative character in general forces us to make use of approximate models in order to understand the strong interaction effects at a small momentum scale. One of these models became known as the global color model (GCM) [1-3], which is a quark-gluon quantum field theory that describes QCD for low-energy processes. There are many recent calculations exemplifying the remarkable success of this procedure [4,5]. It relates the hadronic properties to the Schwinger functions of quarks and gluons. Therefore, when comparing the theoretical calculations to some low-energy data, e.g., pseudoscalars masses and decay constants or other chiral parameters, we learn about the infrared behavior of the quark and gluon propagators. In the near future, this semiphenomenological tool may reveal to be even more successful than the relativistic quark model or the bag model.

In order to have an idea of what is behind the GCM, we can recall that its action is obtained from the QCD generating functional in the standard way [1], with the main difference being that in the functional generator of the connected gluon $n$-point functions we neglect the higher than two $n$-point functions, expecting that a phenomenological description of the gluon propagator $g^{2} D_{\mu \nu}(x-y)$ retains most of the information about the non-Abelian character of QCD. The effect of this approximation can only be measured in comparison with experiments, and in fact it does work quite well once we model appropriately the infrared behavior of the gluon propagator.

Some of the gluon propagators used in the GCM have a quite enhanced infrared behavior. One example is the introduction of a delta function $\delta(k)$ as prescribed in Ref. [6], which is a confining propagator according to the criterion of absence of real $k^{2}$ poles for the quark propagators [6,7]. Another Ansatz for the gluon two-point function in the infrared is $g^{2} D\left(k^{2}\right)=3 \pi^{2}(\chi / \Delta)^{2} \exp \left(-k^{2} / \Delta\right)$ [8], which was inspired by and approaches the $\delta$ function Ansatz of Ref. [6] for $\Delta \rightarrow 0$, where $\chi$ and $\Delta$ are adjustable parameters. In detailed calculations of chiral parameters more complete Ansätze for the gluon propagators than the above ones have been used, in general including the asymptotic behavior of the gluon propagator as predicted by QCD. In Ref. [5] the following form for the gluon propagator in the Landau gauge was introduced:

$$
g^{2} D_{\mu \nu}(k)=\left\{\delta_{\mu \nu}-\frac{k_{\mu} k_{\nu}}{k^{2}}\right\} D\left(k^{2}\right),
$$

where

$$
\begin{aligned}
D\left(k^{2}\right) & \equiv \frac{g^{2}}{k^{2}\left[1+\Pi\left(k^{2}\right)\right]} \\
& =4 \pi^{2} d\left[4 \pi^{2} m_{t}^{2} \delta^{4}(k)+\frac{1-e^{\left(-k^{2} /\left[4 m_{t}^{2}\right]\right)}}{k^{2}}\right],
\end{aligned}
$$

with $d=12 /\left(33-2 n_{f}\right)$, and $n_{f}=3$ (considering only three quark flavors). The mass scale $m_{t}$ determined in Ref. [5] was interpreted as marking the transition between the perturbative and nonperturbative domains. Another Ansatz is [9]

$$
g^{2} D\left(k^{2}\right)=3 \pi^{2} \frac{\chi^{2}}{\Delta^{2}} \exp \left(-k^{2}\right)+\frac{\alpha_{s}\left(k^{2}\right)}{k^{2}} \mathcal{F}\left(k^{2}\right),
$$

where

$$
\alpha_{s}\left(k^{2}\right)=\frac{4 \pi^{2} d g^{2}}{\ln \left(k^{2} / \Lambda^{2}+\tau\right)},
$$

$\mathcal{F}\left(k^{2}\right)$ is a function chosen differently in the papers of Ref. [9], $\alpha_{s}\left(k^{2}\right)$ describes the QCD running coupling constant where $\tau$ is a parameter adjusted phenomenologically. Notice that in the above expressions the momenta are in Euclidean space. Of course, there are still other variations of these $A n$ sätze [10] and attempts to explain the enhanced behavior in the infrared [11].

In this work we study the bifurcation of the quark selfenergy within the context of the GCM, i.e., we determine the critical coupling constant of the truncated Schwinger-Dyson equation for the quark propagator above which dynamical chiral symmetry breaking occurs using the gluon propagators discussed in the previous paragraph. It is known from analytical and numerical studies of the Schwinger-Dyson equations that dynamical chiral symmetry breaking takes place in QCD when the coupling constant $\left(\alpha_{s}\right)$ is of $O(1)$ [12-14]. These studies have also been performed with the use of effective potentials [15] and corroborated by lattice simula- 
tions [16]. Therefore, it is natural to ask what is the critical coupling in the GCM with the phenomenological gluon propagators proposed in the literature.

We will apply the standard techniques of bifurcation theory as used by Atkinson and collaborators [13,17-19] and verify that for the gluon propagator given by a delta function the chiral symmetry is always broken no matter what "perturbative" propagator is added to the $\delta$. For a propagator of the form given by Eq. (3) we obtain a lower bound on the critical coupling smaller than the one obtained for QCD [17], and evaluate the smallest characteristic number confirming the result obtained with the $\delta$ function propagator in the limit that the gaussian width goes to zero. In Sec. II we discuss chiral symmetry breaking for an infrared gluon propagator similar to Eq. (2) using a bifurcation analysis of the Schwinger-Dyson equations. In Sec. III we analyze the infrared gluon propagator given by a Gaussian form using a different technique. The last section contains our conclusions.

\section{THE CRITICAL COUPLING FOR A CONFINING PROPAGATOR}

The Schwinger-Dyson equation for a massless quark propagator in Minkowski space can be written in the form

$$
\begin{aligned}
S^{-1}(p)= & p-l g^{2} \int \frac{d^{4} q}{(2 \pi)^{4}} D_{\mu \nu}^{a b} \\
& \times(p-q) \Gamma^{\mu}(q, p) \frac{\lambda_{c}^{a}}{2} S(q) \gamma^{\nu} \frac{\lambda_{c}^{b}}{2},
\end{aligned}
$$

where the gluon propagator in the Landau gauge, which will be used throughout the paper, is given by

$$
D_{\mu \nu}^{a b}(k)=\delta^{a b}\left[-g_{\mu \nu}+\frac{k_{\mu} k_{\nu}}{k^{2}}\right] \frac{1}{k^{2}+i \epsilon} .
$$

In the case that the gluon propagator is given by the confining delta function [6]

$$
D_{\mu \nu}^{a b}(k)=\delta^{a b}\left[g_{\mu \nu}-\frac{k_{\mu} k_{\nu}}{k^{2}}\right] \beta \delta^{4}(k),
$$

where $\beta$ is an adjustable dimensional parameter. Note that the term $k_{\mu} k_{\nu} \delta(k) / k^{2}$ is undefined from the point of view of generalized functions. Of course, we could work with the Feynman gauge where this unwanted behavior is softened. Fortunately the $\delta$ function gives an integrable singularity and only because of this peculiarity we have not a strong pathological behavior. With Eq. (7) it is quite easy to verify that the quark self-energy has a nontrivial solution for any positive value of $g^{2}$, in the rainbow approximation $\left[\Gamma^{\mu}(q, p)\right.$ $\left.=\gamma^{\mu}\right]$. With the quark propagator given by

$$
S^{-1}(p)=A\left(p^{2}\right) p-B\left(p^{2}\right),
$$

and the gluon propagator of Eq. (7) it follows from Eq. (5), in Euclidean space, the set of coupled integral equations

$$
\begin{aligned}
{\left[A\left(p^{2}\right)-1\right] p^{2}=} & \frac{4}{3} g^{2} \beta \int \frac{d^{4} q}{(2 \pi)^{4}} \delta^{4}(p-q) \\
& \times \frac{A\left(q^{2}\right)}{q^{2} A^{2}\left(q^{2}\right)+B^{2}\left(q^{2}\right)} \\
& \times\left[p q+2 \frac{q(p-q)(p-q) p}{(p-q)^{2}}\right], \\
B\left(p^{2}\right)= & 4 g^{2} \beta \int \frac{d^{4} q}{(2 \pi)^{4}} \delta^{4}(p-q) \\
& \times \frac{B\left(q^{2}\right)}{q^{2} A^{2}\left(q^{2}\right)+B^{2}\left(q^{2}\right)} .
\end{aligned}
$$

The solutions of the nonlinear coupled integral equations were determined in Refs. [1,2], showing that $A\left(p^{2}\right)$ is a constant. Without loss of generality we can set $A\left(p^{2}\right)=1$ and verify the behavior of the self-energy apart from a normalization constant. In this case we obtain the following equation for $B\left(p^{2}\right)$

$$
B\left(p^{2}\right)=\frac{4 g^{2} \beta}{(2 \pi)^{4}} \int d^{4} q \frac{B\left(q^{2}\right) \delta^{4}(q-p)}{q^{2}+B^{2}\left(q^{2}\right)},
$$

whose integration leads to

$$
B\left(p^{2}\right)=\frac{4 g^{2} \beta}{(2 \pi)^{4}} \frac{B\left(p^{2}\right)}{p^{2}+B^{2}\left(p^{2}\right)} .
$$

Equation (11) has the solution

$$
B\left(p^{2}\right)=\left\{\begin{array}{l}
\sqrt{\frac{4 g^{2} \beta}{(2 \pi)^{4}}-p^{2}}, \quad p^{2} \leqslant \frac{4 g^{2} \beta}{(2 \pi)^{4}}, \\
0, \quad p^{2}>\frac{4 g^{2} \beta}{(2 \pi)^{4}},
\end{array}\right.
$$

for any nonzero and positive value of the coupling constant. If we consider only this infrared behavior of the gluon propagator we would conclude that this model is inconsistent with previous work on QCD, which predicts a critical value of the coupling constant for the onset of chiral symmetry breaking. However, the ultraviolet part of the gluon propagator cannot be neglected and the use of the following propagator is frequent $[2,5]$

$$
D_{\mu \nu}^{a b}(k)=\delta^{a b}\left[\delta_{\mu \nu}-\frac{k_{\mu} k_{\nu}}{k^{2}}\right] \beta \delta^{4}(k)+\delta^{a b}\left[\delta_{\mu \nu}-\frac{k_{\mu} k_{\nu}}{k^{2}}\right] \frac{1}{k^{2}} .
$$

The following comments are in order: (a) In some papers the coupling constant $g^{2}$ is absorbed in the parameter $\beta$ [5]. Here we assume that $g^{2}$ can always be factorized, and the critical behavior is going to be related to it. (b) We assume a constant $g^{2}$. In the next section it will be shown that our result does not change with the inclusion of the running coupling in the ultraviolet part of the gluon propagator. (c) This propagator has been used in the literature with some minor 
differences; these variations are not important for our result as will become clear in the next section.

When the gluon propagator is given by Eq. (12) we need a thorough analysis of the quark self-energy to find for which value of the coupling constant the quark self-energy admits a nontrivial solution. The substitution of Eq. (12) into Eq. (5) yields

$$
B(x)=\frac{g^{2}}{4 \pi^{2}}\left(\frac{\kappa B(x)}{x+B^{2}(x)}+\int_{\mu^{2}}^{\Lambda^{2}} \frac{d y}{x_{\max }} \frac{y B(y)}{y+B^{2}(y)}\right),
$$

where $x=p^{2}, y=q^{2}, x_{\max }=\max (x, y)$, and $\kappa=\beta / \pi^{2}$. We will be looking for solutions of Eq. (13) only in the interval $\left[\mu^{2}, \Lambda^{2}\right]$, although the $\delta$ function has been integrated in the full range of momenta. A rigorous handling of this integration does not change the conclusions about the bifurcation point in the limit of very small (large) infrared (ultraviolet) cutoff.

It is important to verify that Eq. (13) is a very peculiar one, in the sense that for very small values of $g^{2}$, keeping the product $g^{2} \kappa$ fixed, we do have the solution of the nonlinear equation, which, at low momenta, approaches

$$
B(x \rightarrow 0)=\left(\sqrt{\frac{g^{2} \kappa}{4 \pi^{2}}}\right)_{g^{2} \rightarrow 0, g^{2} \kappa \text { fixed }} .
$$

To find nontrivial small solutions of the nonlinear equation (13) we study the linearized equation, i.e., the functional derivative of Eq. (13) evaluated at $B(x)=0 \quad[13,17-19]$. Writing $\delta B=f$, we obtain

$$
f(x)=\frac{g^{2}}{4 \pi^{2}}\left(\frac{\kappa f(x)}{x}+\int_{\mu^{2}}^{\Lambda^{2}} d y \frac{f(y)}{x_{\max }}\right) .
$$

Note that we are not considering the equation for $A\left(p^{2}\right)$ because this equation is of second order in $\delta B$ [19]. The existence of a solution for Eq. (15) is a sufficient condition for the onset of chiral symmetry breaking [13]. Defining $\alpha$ $\equiv g^{2} / 4 \pi^{2}=\alpha_{s} / \pi$, Eq. (15) is equivalent to the differential equation

$$
x(x-\alpha \kappa) f^{\prime \prime}(x)+2 x f^{\prime}(x)+\alpha f(x)=0,
$$

together with the ultraviolet boundary condition $[(x$ $-\alpha \kappa) f(x)]\left.^{\prime}\right|_{x \rightarrow \Lambda^{2}}=0$ and another condition valid in the infrared region.

The choice of the infrared boundary condition is a crucial one. Note that if we neglect the second term of the righthand side of Eq. (13) we only obtain a trivial result, no matter what is the boundary condition coming from Eq. (13). However, we know that the nonlinear equation always has a nontrivial solution for the $\delta$ function propagator. The most suitable infrared boundary condition is the one that comes from the nonlinear integral equation (12), since when applied to the linear equation the result must be consistent with the known solution of the nonlinear equation for small $g^{2}$ [see Eq. (14)]. Therefore, our infrared boundary condition is given by

$$
\left.f(x)\right|_{x \rightarrow \mu^{2}}=\sqrt{\alpha \kappa} .
$$

Equation (16) can be put in the form of a hypergeometric equation performing a shift $x \rightarrow z+\alpha \kappa$ and defining $y=$ $-z / \alpha \kappa$ leading to

$$
\begin{aligned}
& y(1-y) f^{\prime \prime}(-\alpha \kappa y+\alpha \kappa) \\
& \quad+2(1-y) f^{\prime}(-\alpha \kappa y+\alpha \kappa)+\alpha f(-\alpha \kappa y+\alpha \kappa)=0,
\end{aligned}
$$

The solution of Eq. (18) that obeys the infrared boundary condition is

$$
f(x)=A F\left(a, b ; 2 ; 1-\frac{x}{\alpha \kappa}\right),
$$

where

$$
A=\sqrt{\alpha \kappa} \Gamma(3 / 2-\sigma) \Gamma(3 / 2+\sigma),
$$

is a normalization constant, $\sigma$ is defined as $\sigma=\left(\frac{1}{4}-\alpha\right)^{1 / 2}$, and

$$
\begin{aligned}
& a=\frac{1}{2}+\sqrt{\frac{1}{4}-\alpha}, \\
& b=\frac{1}{2}-\sqrt{\frac{1}{4}-\alpha} .
\end{aligned}
$$

To apply the ultraviolet boundary condition we recall the following relation involving hypergeometric functions:

$$
\frac{d}{d z}\left[z^{c-1} F(a, b ; c ; z)\right]=(c-1) z^{c-2} F(a, b ; c-1 ; z),
$$

which lead us to the analysis of the zeros of the following equation:

$$
\begin{aligned}
& {\left.[(x-\alpha \kappa) f(x)]^{\prime}\right|_{x \rightarrow \Lambda^{2}}} \\
& \quad=-\left.\alpha \kappa A F\left(1 / 2+\sigma, 1 / 2-\sigma ; 1,1-\frac{x}{\alpha \kappa}\right)\right|_{x \rightarrow \Lambda^{2}} .
\end{aligned}
$$

We need to consider the solution of Eq. (23) in three different regions of the parameter $\alpha$, namely, $0<\alpha<\frac{1}{4}, \alpha$ $=\frac{1}{4}$, and $\alpha>\frac{1}{4}$, and study the asymptotic behavior of $f(x)$ in each one of these cases. When $\alpha<\frac{1}{4}$ the relation 


$$
\begin{aligned}
& F(a, b ; c ; z) \\
& =\frac{\Gamma(c) \Gamma(b-a)}{\Gamma(b) \Gamma(c-a)}(-z)^{-a} F\left(a, 1+a-c ; 1+a-b ; z^{-1}\right) \\
& \quad+\frac{\Gamma(c) \Gamma(a-b)}{\Gamma(a) \Gamma(c-b)}(-z)^{-b} F\left(1+b-c, b ; 1+b-a ; z^{-1}\right),
\end{aligned}
$$

can be used together with

$$
F(a, b ; c ; 0)=1
$$

to give

$$
\begin{aligned}
& {\left.[(x-\alpha \kappa) f(x)]^{\prime}\right|_{x \rightarrow \Lambda^{2}}} \\
& \quad=A^{\prime}\left(\frac{x}{\alpha \kappa}-1\right)^{-1 / 2+\sigma}+\left.B^{\prime}\left(\frac{x}{\alpha \kappa}-1\right)^{-1 / 2-\sigma}\right|_{x \rightarrow \Lambda^{2}},
\end{aligned}
$$

where $A^{\prime}$ and $B^{\prime}$ depend on $\sigma$ and $\alpha \kappa$.

In this case $\sigma$ is a real and positive number smaller than $\frac{1}{2}$. For large values of $x$ we see that Eq. (26) condition is satisfied. Therefore, Eq. (19) is a solution when $0<\alpha<\frac{1}{4}$.

Note that when $\alpha=\frac{1}{4}$ we have $\sigma=0$, the constants $a$ and $b$ are identical, implying that Eq. (24) cannot be used in this case. However, we can perform the limit $x \rightarrow \Lambda^{2}$ already in the differential equation (16) and study its behavior for this particular value of $\alpha$. In the asymptotic region (UV) we obtain

$$
f(x)_{U V}=x^{-1 / 2}(C+D \ln x) .
$$

We easily see that the ultraviolet boundary condition is satisfied for large $x$ and Eq. (19) is also a solution when $\alpha=\frac{1}{4}$.

When $\alpha>\frac{1}{4}$ in the parameters $a$ and $b$ of Eq. (19) we make the substitution $\sigma \rightarrow i \rho$ with $\rho$ defined as $\left(\alpha-\frac{1}{4}\right)^{1 / 2}$. The asymptotic behavior in this case is obtained with the help of Eqs. (25) and (24) leading to

$$
\begin{aligned}
{\left.[(x-\alpha \kappa) f(x)]^{\prime}\right|_{x \rightarrow \Lambda^{2}} } & -2(\alpha \kappa)^{3 / 2} \operatorname{Re}\left[\frac{\Gamma(2 i \rho) \Gamma(3 / 2-\sigma) \Gamma(3 / 2+\sigma)}{\Gamma^{2}(1 / 2+i \rho)}\right] \\
& \times\left.\left(\frac{x}{\alpha \kappa}-1\right)^{-1 / 2+i \rho}\right|_{x \rightarrow \Lambda^{2}}
\end{aligned}
$$

which is valid for $x \gg \alpha \kappa$. Equation (28) has an infinite set of zeros located at $x=\alpha \kappa\left(x_{n}+1\right)$ for integer $n$, which can be determined with the procedure of the second paper of Ref. [13].

The real part of Eq. (28) can be written as

$$
\exp \left\{\ln \left[\frac{\Gamma(2 i \rho) \Gamma(3 / 2-\sigma) \Gamma(3 / 2+\sigma)}{\Gamma^{2}(1 / 2+i \rho)} x_{n}^{-1 / 2+i \rho}\right]\right\},
$$

as $\ln z=\ln |z|+i(\arg z \pm 2 n \pi)$, where $z$ is a complex number and $n=0,1,2, \ldots$, the zeros of this function will occur for

$$
\ln \left(x_{n}\right) \sim \frac{1}{\rho}\left[2 n \pi-\arg \left(\frac{\Gamma(2 i \rho) \Gamma(3 / 2-\sigma) \Gamma(3 / 2+\sigma)}{\Gamma^{2}(1 / 2+i \rho)}\right)\right]
$$

with $n=1,2, \ldots$. This relation was obtained for $x \gg \alpha \kappa$, and $n=0$ was excluded in order to obtain only positive values in Eq. (30). Therefore, also for $\alpha>\frac{1}{4}$ we do have a nontrivial solution.

In summary, when the gluon propagator is modeled by a delta function plus a "perturbative", $1 / k^{2}$ propagator we verified that the chiral symmetry is broken for any positive value of the coupling constant. One may ask if this result changes if we modify the nonperturbative as well as the perturbative propagator, as in the case of Eq. (2), or with the inclusion of the running coupling or the effect of a dynamical gluon mass $[13,20]$. We will show in the next section that this is not the case.

\section{AN INFRARED MODEL IN THE GAUSSIAN FORM}

As discussed in the introduction a different Ansatz for the gluon propagator, exemplified by Eq. (3), has frequently been used. Its nonperturbative infrared behavior is given by the first term of Eq. (3):

$$
D_{\mu \nu}^{a b}(k)=\delta^{a b}\left[\delta_{\mu \nu}-\frac{k_{\mu} k_{\nu}}{k^{2}}\right] 3 \pi^{2} \frac{\chi^{2}}{\Delta^{2}} \exp \frac{-k^{2}}{\Delta}
$$

where the parameter $\chi$ controls the intensity of the interaction, and $\Delta$ gives the Gaussian width. It is important to note that in the limit $\Delta \rightarrow 0$ we recover the infrared behavior of the propagator discussed in the previous section.

In this section we will show that the quark self-energy calculated with the propagator of Eq. (31) has a well defined bifurcation point. Afterwards, we verify that adding a perturbative tail to this propagator results in a smaller critical coupling constant than the one obtained only with the perturbative part. Finally, in the limit $\Delta \rightarrow 0$, we recover the result of the previous section showing that it is independent of the perturbative part that is added to the delta function.

To find nontrivial small solutions of the nonlinear Schwinger-Dyson equation for the quark self-energy we can study the linearized equation. The substitution of Eq. (31) into Eq. (5) gives the following linear integral equation for $f(x)$ [recalling that $f(x)=\delta B(x)]$ :

$$
f(x)=\frac{3}{4} g^{2} \frac{\chi^{2}}{\Delta^{2}}\left[\int_{0}^{x} d y f(y) \exp \frac{-x}{\Delta}+\int_{x}^{\infty} d y f(y) \exp \frac{-y}{\Delta}\right],
$$

where we considered $A\left(p^{2}\right)=1$, since, according to bifurcation theory, the equation for $\left[A\left(p^{2}\right)-1\right]$ is of higher order in the functional derivative of $B\left(p^{2}\right)$, and the critical coupling constant is determined only through Eq. (32). To obtain Eq. (32) we performed in the gluon propagator the so-called angle approximation, which is given by 


$$
D\left[(p-q)^{2}\right] \approx \theta\left(p^{2}-q^{2}\right) D\left(p^{2}\right)+\theta\left(q^{2}-p^{2}\right) D\left(q^{2}\right) .
$$

The Eq. (32) is a homogeneous Fredholm equation with the kernel

$$
K_{I}(x, y)=\exp \left(-\frac{x}{\Delta}\right) \theta(x-y)+\exp \left(-\frac{y}{\Delta}\right) \theta(y-x) .
$$

The norm of Eq. (34) is easily calculated

$$
\left\|K_{I}\right\|^{2}=\int_{0}^{\infty} d x \int_{0}^{\infty} d y K_{I}^{2}(x, y)=\frac{\Delta^{2}}{2},
$$

and we find nontrivial $L^{2}$ solutions of $B(x)$ for $g^{2}$ on a point set whose smallest positive point satisfies [17]

$$
g^{2} \geqslant \frac{4 \Delta^{2}}{3 \chi^{2}} \frac{1}{\left\|K_{I}\right\|}=\left(\frac{4 \sqrt{2}}{3}\right) \frac{\Delta}{\chi^{2}} \approx 1.88 \frac{\Delta}{\chi^{2}} .
$$

Equation (36) gives a lower bound for the critical point $g_{c}^{2}$. However, we can do better than this. Using the method of traces we can show that the approximate critical value is indeed of the order of the smaller value of the bound given by Eq. (36).

The following approximate formula holds true for the smallest characteristic number $g_{c}^{2}$ [21]:

$$
\left|g_{c}^{2}\right| \approx \sqrt{\frac{A_{2}}{A_{4}}}
$$

where, for a symmetric kernel,

$$
A_{2 m}=2 \int_{0}^{\infty} \int_{0}^{x} K_{m}^{2}(x, y) d y d x,
$$

with $m$ running over $1,2 . K_{2}(x, y)$ is given by

$$
K_{2}(x, y)=\int_{0}^{\infty} K_{1}(x, z) K_{1}(z, y) d z,
$$

and, in the case of the infrared part of the propagator, $K_{1}(x, y)=\left(3 \chi^{2} / 4 \Delta^{2}\right) g^{2} K_{I}(x, y)$. The calculation of Eq. (37) entails

$$
g_{c}^{2} \approx \sqrt{\frac{9 \chi^{4} / 32 \Delta^{2}}{297 \chi^{8} / 4096 \Delta^{4}}} \approx 1.97 \frac{\Delta}{\chi^{2}} .
$$

It is known that this method overestimates $g_{c}^{2}$. In this case we verify that the smallest value of our lower bound Eq. (36) is a good approximation for the critical point. The value of the critical coupling can be obtained substituting the phenomenological values of $\chi$ and $\Delta$ into Eq. (36). It is obvious that in the limit $\Delta \rightarrow 0$ we recover the result of the previous section, i.e., with the gluon propagator given by a $\delta$ function the chiral symmetry is broken for any coupling constant $g^{2}$ $>0$.
Let us now consider the case where we add to the Gaussian form of the infrared propagator a perturbative contribution. If this new contribution is of the form given by Eq. (2) or (3), it is not difficult to verify the existence of a critical coupling constant for the onset of chiral symmetry breaking. The reason for this is that the ultraviolet behavior of the propagator is softened by the factors $1-\exp \left(-k^{2} /\left[4 m_{t}^{2}\right]\right)$ and $\mathcal{F}\left(k^{2}\right)$, producing an effect equivalent to a dynamical gluon mass [10] for which it was shown the existence of a bifurcation point $[13,20]$. Therefore, we proceed to the most intriguing case where we add to the Ansatz for the infrared gluon propagator the contribution of QCD with massive gluons and the effect of the running coupling constant, i.e., a propagator proportional to $\alpha_{s}\left(k^{2}\right) /\left(k^{2}+m_{g}^{2}\right)$.

If we denominate by $K_{U}$ the kernel related to this ultraviolet propagator, we assume that we do have a nontrivial solution for

$$
g^{2} \geqslant \frac{1}{\left\|K_{U}\right\|}
$$

A lower bound for the bifurcation point in the case that we consider the sum of propagators will be given by

$$
g_{c}^{2}\left\|\frac{3 \chi^{2}}{4 \Delta^{2}} K_{I}+K_{U}\right\| \geqslant 1,
$$

where $\left\|K_{I}\right\|$ is given by Eq. (35). With the triangle inequality

$$
\left\|K_{A}+K_{B}\right\| \leqslant\left\|K_{A}\right\|+\left\|K_{B}\right\|,
$$

and making use of Eq. (35) we obtain

$$
g^{2}\left(\frac{3 \sqrt{2}}{8} \frac{\chi^{2}}{\Delta}+\left\|K_{U}\right\|\right) \geqslant 1 .
$$

The solutions that break the chiral symmetry appear only for values of the coupling constant obeying Eq. (44). Note that as long as we can factor out $g^{2}$ from both propagators, and as long as the kernel of the perturbative tail is bounded, we obtain a condition for values of $g^{2}$ above which the selfenergy bifurcates. The lower bound for the critical value

$$
g_{c}^{2} \geqslant \frac{1}{\left[(3 \sqrt{2} / 8)\left(\chi^{2} / \Delta\right)+\left\|K_{U}\right\|\right]},
$$

approaches zero as we take the limit $\Delta \rightarrow 0$, and is compatible with the result of the previous section.

Considering the infrared and ultraviolet contributions to the propagator, we apply again the method of traces to determine the critical coupling constant through Eqs. (37) and (38). In this case the kernel $K_{1}(x, y)$ in Eq. (38) is given by

$$
K_{1}(x, y)=H(x) \theta(x-y)+H(y) \theta(y-x),
$$

where

$$
H(x)=\frac{3 \chi^{2}}{4 \Delta^{2}} \exp \left(\frac{-x}{\Delta}\right)+\frac{1}{x+m_{g}^{2}} \frac{4 \pi^{2} d}{\ln \left(x / \Lambda^{2}+\tau\right)} .
$$




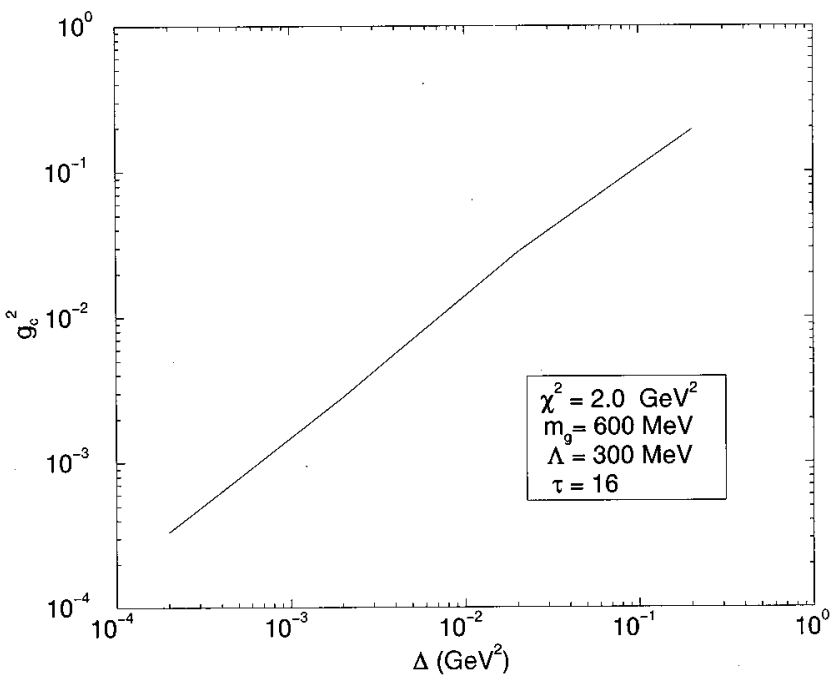

FIG. 1. Critical coupling constant, $g_{c}^{2}$, as a function of the parameter $\Delta$, considering the infrared and ultraviolet contributions to the gluon propagator. We see that decreasing the value of $\Delta$ the critical coupling goes to zero. The evaluation was performed for $n_{f}=4, \chi^{2}=2.0 \mathrm{GeV}^{2}, m_{g}=600 \mathrm{MeV}, \Lambda=300 \mathrm{MeV}$, and $\tau=16$.

Note that we have already factored out from the kernel the coupling $g^{2}$. To determine $A_{4}$ we need $K_{2}(x, y)$ which is equal to

$$
\begin{aligned}
K_{2}(x, y)= & H(x) H(y) \int_{0}^{y} d z+H(x) \int_{y}^{x} d z H(z) \\
& +\int_{x}^{\infty} d z H^{2}(z) .
\end{aligned}
$$

The critical coupling constant was determined numerically for four quark flavors, $\chi^{2}=2.0 \mathrm{GeV}^{2}, m_{g}=600 \mathrm{MeV}$, $\Lambda=300 \mathrm{MeV}$, and we assumed $\tau=16$, where all these values are phenomenologically acceptable, and, in particular, the value of $\tau$ is compatible with the infrared behavior of the running coupling constant in a theory admitting dynamically generated gluon masses [22] [in these papers we have $\tau$ $\left.\approx 4\left(m_{g}^{2} / \Lambda^{2}\right)\right]$. The critical coupling is shown in Fig. 1 as a function of the parameter $\Delta$. Note that as we decrease $\Delta$ the value of the critical coupling goes to zero, confirming the result of the previous section. In this region also the integrals appearing in Eq. (48) become more problematic because the Gaussian becomes very peaked. Changes in the form of the ultraviolet propagator barely affect this result. Finally, we confirm that for this class of infrared propagators the chiral phase transition happens at a smaller value than the one obtained with perturbative QCD.

\section{CONCLUSIONS}

An Ansatz for the infrared gluon propagator in the form of a $\delta^{4}(q)$ function (or a variation thereof), frequently used in applications of the global color model, gives an excellent description of hadronic properties connected with chiral symmetry breaking. In this work we studied the bifurcation of the quark self-energy within this model. The idea was to compare GCM to QCD with just the perturbative gluon propagator, where it is known that above a certain critical value of the coupling constant the chiral symmetry is broken [13-15]. We verified that the introduction of a delta function to describe the infrared behavior of the gluon propagator implies that the chiral symmetry is always broken for any value of the coupling constant. This is an interesting result if we remember that this propagator is considered to describe confined quarks [6]. However, this is certainly in contrast with what is known to happen in QCD.

With a model for the gluon propagator inspired by the delta function but softer at the origin (in a Gaussian form), we verified, using the method of traces, that the critical coupling for the onset of chiral symmetry breaking is lower than the one expected for perturbative QCD, and recovered in a particular limit the result obtained with the model involving a delta function for the infrared gluon propagator. Therefore, the critical point for chiral symmetry breaking may distinguish among different QCD motivated models, and in the cases we have studied the critical coupling is always smaller than the one known for QCD $[14,15]$.

\section{ACKNOWLEDGMENTS}

We would like to thank E.V. Gorbar for valuable remarks. This research was supported by the Conselho Nacional de Desenvolvimento Científico e Tecnológico (CNPq) (A.A.N., R.R.), by Fundacão de Amparo à Pesquisa do Estado de São Paulo (FAPESP) (A.C.A., A.A.N., R.R.) and by Programa de Apoio a Núcleos de Excelência (PRONEX).
[1] P. C. Tandy, Prog. Part. Nucl. Phys. 39, 117 (1997).

[2] C. D. Roberts and A. G. Williams, Prog. Part. Nucl. Phys. 33, 477 (1994).

[3] R. T. Cahill and S. M. Gunner, Fiz. B 7, 171 (1998).

[4] C. D. Roberts, R. T. Cahill, M. Sevior, and N. Iannella, Phys. Rev. D 49, 125 (1994); C. D. Roberts, ANL Report No. ANLPHY-7842-TH-94, 1994; in Chiral Dynamics: Theory and Experiment, Proceedings of the Workshop, MIT, 1994, Lecture Notes in Physics No. 452 (Springer-Verlag, New York, 1995), p. 68; K. L. Mitchell, P. C. Tandy, C. D. Roberts, and R. T. Cahill, Phys. Lett. B 335, 282 (1994); M. R. Frank, K. L.
Mitchell, C. D. Roberts, and P. C. Tandy, ibid. 359, 17 (1995); C. J. Burden, Lu Qian, C. D. Roberts, P. C. Tandy, and M. J. Thomson, Phys. Rev. C 55, 2649 (1997).

[5] M. R. Frank and C. D. Roberts, Phys. Rev. C 53, 390 (1996).

[6] H. J. Munczek and A. M. Nemirovsky, Phys. Rev. D 28, 181 (1983); D. W. McKay and H. J. Munczek, ibid. 55, 2455 (1997).

[7] H. Pagels, Phys. Rev. D 14, 2747 (1976); 15, 2991 (1977).

[8] L. S. Kisslinger and T. Meissner, Phys. Rev. C 57, 1528 (1998).

[9] M. R. Frank and T. Meissner, Phys. Rev. C 53, 2410 (1996); P. 
Maris and P. C. Tandy, ibid. 60, 055214 (1999); L. S. Kisslinger, M. Aw, A. Harey, and O. Linsuain, ibid. 60, 065204 (1999).

[10] A. A. Natale and P. S. Rodrigues da Silva, Phys. Lett. B 442, 369 (1998).

[11] H.-P. Pavel, D. Blaschke, V. N. Pervushin, and G. Röpke, Int. J. Mod. Phys. A 14, 205 (1999); A. A. Natale, Mod. Phys. Lett. A 14, 2049 (1999).

[12] P. I. Fomin, V. P. Gusynin, V. A. Miransky, and Yu. A. Sitenko, Riv. Nuovo Cimento 6, 1 (1983); V. A. Miransky, Sov. J. Nucl. Phys. 38, 280 (1983); Phys. Lett. 165B, 401 (1985); K. Higashijima, Phys. Rev. D 29, 1228 (1984).

[13] D. Atkinson and P. W. Johnson, Phys. Rev. D 35, 1943 (1987); 37, 2290 (1988); J. Math. Phys. 28, 2488 (1987).

[14] D. Atkinson and P. W. Johnson, Phys. Rev. D 37, 2296 (1988); C. D. Roberts and B. H. J. McKellar, ibid. 41, 672 (1990).

[15] M. E. Peskin, in Recent Advances in Field Theory and Statistical Mechanics, edited by J.-B. Zuber and R. Stora (Elsevier
Science, British Vancouver, 1984), p. 217; A. A. Natale, Nucl. Phys. B226, 365 (1983); A. Barducci et al., Phys. Rev. D 38, 238 (1988).

[16] J. Kogut, M. Stone, H. W. Wyld, J. Shigemitsu, S. H. Shenker, and D. K. Sinclair, Phys. Rev. Lett. 48, 1140 (1982).

[17] D. Atkinson, J. Math. Phys. 28, 2494 (1987).

[18] D. Atkinson, P. W. Johnson, and K. Stam, Phys. Lett. B 201, 105 (1988).

[19] D. Atkinson, V. P. Gusynin, and P. Maris, Phys. Lett. B 303, 157 (1993).

[20] A. A. Natale and P. S. Rodrigues da Silva, Phys. Lett. B 392, 444 (1997).

[21] S. G. Miklin, Integral Equations, Vol. 04 of International Series of Monographs in Pure and Applied Mathematics (Pergamon Press, London, 1957).

[22] J. M. Cornwall, Phys. Rev. D 26, 1453 (1982); J. M. Cornwall and J. Papavassiliou, ibid. 40, 3474 (1989); 44, 1285 (1991). 